\title{
ARTICLE
}

\section{Alcohol misuse in older people}

\author{
Rahul Rao \& Ilana Crome
}

Rahul Rao is a consultant old age psychiatrist with South London and Maudsley NHS Foundation Trust and a visiting researcher at Institute of Psychiatry, Psychology \& Neuroscience (loPPN), King's College London. He has both clinical and research interests in alcohol-related mental disorders, also working in an area with a high rate of alcohol misuse in older people. He chairs the Substance Misuse in Older People Working Group at the Royal College of Psychiatrists. Ilana Crome is an Emeritus Professor at Keele University, Honorary Professor at Queen Mary University London, and Visiting Professor at St George's, University of London. Professor Crome specialises in addiction across the lifespan. Her research interests centre on epidemiology, comorbid mental health and addictive disorders, distinctive problems in young and older substance misusers, and professional education in substance misuse. Correspondence Dr Rahul Rao, Southwark Community Team for Older People, South London and Maudsley NHS Foundation Trust, Ground Floor, 63-65 Marina House, Denmark Hill, London SE5 8RS, UK. Email: tony.rao@slam.nhs.uk

\begin{abstract}
SUMMARY
The clinical and public mental health aspects of alcohol misuse in older people (both men and women) have increasing relevance for both old age and addiction psychiatrists. Clinical presentations are often complex and involve a number of different psychiatric, physical and psychosocial factors. The assessment, treatment and aftercare of alcohol-related and comorbid other mental disorders will also involve a broad range of interventions from a wide range of practitioners. Given its growing clinical relevance, there are particular areas, such as alcohol-related brain damage and drug interactions with alcohol, that deserve special attention.
\end{abstract}

\section{LEARNING OBJECTIVES}

- Know about assessment, psychosocial intervention and recovery in alcohol misuse in older people, and the related legal/ethical framework

- Understand the role of physical and psychiatric illness in the presentation of older people with alcohol misuse

- Give due consideration to the general management of alcohol problems in older people, with special consideration of drug interactions and alcohol-related brain damage

\section{DECLARATION OF INTEREST}

None

In 2011, the Royal College of Psychiatrists published its first report on substance misuse and older people, Our Invisible Addicts (Crome 2011). This set out key recommendations at the levels of policy, public health, service delivery and treatment, as well as training and education. Translating these needs into guidance is a key step in the process of achieving integrated care for older people with alcohol misuse and comorbid psychiatric disorder (Rao 2015a). This article details the rationale for nurturing this area of clinical practice in psychiatry by expanding on the main areas for development, with a clinical focus. These areas comprise the epidemiology of alcohol misuse in older people; assessment (including screening); psychosocial interventions; supporting families and carers; legal and ethical aspects; acute psychiatric presentations; drug interactions; alcohol-related brain damage; and the relevance of multi-agency working.

\section{Epidemiology}

The past decade has seen a sharp escalation in morbidity and mortality from alcohol misuse in older people. Between 2005 and 2013, the percentage of men in the UK drinking 8 or more units of alcohol on any one day in the past week fell by only $5 \%$ in those aged 65 and over, compared with a reduction of at least $12 \%$ in all other age groups (Office for National Statistics, 2013). The highest mortality rate for alcoholrelated deaths was in men aged between 55 and 74 (Crome 2011). Over the past 10 years, the number of people between the ages of 60 and 74 admitted to hospitals in England with mental and behavioural disorders associated with alcohol use has risen by over 50\% more than in the 15-59 age group. People aged 75 and over with mental and behavioural disorders associated with alcohol experienced longer hospital admissions than their younger counterparts (Alcohol Concern 2013a). In addition, the number of people aged 60 and over admitted to hospitals in England with amnestic syndrome has risen by over $140 \%$ over the past 10 years, compared with an almost static rise in the 15-59 age group (Alcohol Concern 2013a).

These overall findings cannot be explained purely by rising numbers of older people in the general population, given that the population of people aged 65 and above in England and Wales increased by 11\% between 2001 and 2011 (Office for National Statistics 2012).

\section{Assessment}

The assessment of an older person with alcohol misuse requires careful consideration (Box 1), taking into account age-specific factors that may influence the approach to interviewing, clinical presentation and risks from the misuse. It is important to recognise that even low levels of alcohol use in older people can be harmful, so that dependence criteria do not necessarily have to be met to form a judgement as to whether alcohol is partly or wholly responsible for the clinical presentation.

In late-onset drinking, the bias is towards women, whose problem drinking in later life is usually in reaction to a life crisis (Schonfeld 1991). Women are a particularly vulnerable group, as alcohol misuse is associated with depression and 
social isolation, both of which are more common in older women than in older men (Van den Berg 2014).

\section{Screening for alcohol misuse}

The CAGE questionnaire (Ewing 1984) screens for the core features of alcohol dependence, but it is insensitive to harmful and hazardous drinking in older people. The Alcohol Use Disorders Identification Test (AUDIT) has been validated in some older populations (Roberts 2005), with greatest sensitivity and specificity being shown with a cutoff point of 5 for older men and 3 for older women.

Shorter versions of the AUDIT include the AUDIT-5 (5 items) and the AUDIT-C (3 items). A cut-off point of 4 has been suggested for both the AUDIT-5 (Philpot 2003) and the AUDIT-C (Aalto 2011). However, these studies have not undergone extensive replication in older people. The Short Michigan Alcoholism Screening Test - Geriatric version (SMAST-G) (Blow 1998) has shown the greatest validity and use in older populations.

Given the lack of sensitive screening tools for alcohol problems in older people, tools for working-age adults need to be combined with quantity/frequency measures and a comprehensive assessment that incorporates the approach taken in Box 1.

\section{Psychosocial interventions}

Only $6-7 \%$ of high-risk substance misusers over the age of 60 receive the treatment that they require (Alcohol Concern 2013b). However, since older people are likely to be in contact with the healthcare system, there are significant opportunities to identify problems associated with substance misuse.

Recent studies have shown positive outcomes from psychosocial interventions. Although there is relatively little research in this age group, consistently positive findings emerged from those studies in which psychosocial treatment for alcohol problems in the older patient was investigated. The studies demonstrated that older people want to abstain; have the capacity to change; can be successfully offered help by physicians; respond well to brief advice and motivational enhancement therapy; do not necessarily need age-specific treatment programmes; can achieve improvement in outcomes across a range of domains (mental and physical health, relationships, legal, occupational and financial issues) comparable to that in younger adult populations; and have the prospect of longterm recovery. Although more research needs to be done, older adults should not be barred from treatment because of age.
B0X 1 Assessment of an older person with alcohol misuse

\section{General principles}

- Respect dignity, individuality, values and experiences

- Take into account sensory and cognitive impairment

- Be aware that atypical presentations and underreporting are common

- Use additional information from other sources

- Be aware of psychiatric comorbidity, functional abilities and loss events

- Consider the influence of other substances, physical disorders and social support

Special areas for consideration

- Living arrangements

- Other substance use and misuse (nicotine/over-thecounter/prescribed/illicit drugs)

- Access to alcohol (e.g. relatives/formal carers/home delivery)

- Drinking 'environment' (e.g. home drinking, drinking partners)

- Medical history (physical complications from alcohol)

- Mental capacity

- Drug interactions (including other substances)

- Risk of falls, social/cultural isolation and elder abuse (including need for safeguarding vulnerable adults)

- Level of nutrition

- Social support from informal carers and friends

- Social pressures from debt and alcohol-using carers

- Comorbid psychiatric illness (mostly depression and alcohol-related brain damage)

The Brief Intervention and Treatment for Elders (BRITE) project (Schonfeld 2010) reported a reduction in alcohol use and problems from 80 to $18 \%$, but there was no control group in this study. The Healthy Living as You Age (HLAYA) study found improvement in both controls (advice only) and intervention (integrated care) groups at 12 months (Borok 2013). This is in keeping with the Primary Care Research in Substance Abuse and Mental Health for the Elderly (PRISM-E) study, which found that patients did better in integrated mental health and substance misuse care in primary care compared with referral to specialist providers (Oslin 2006).

Box 2 outlines some of the challenges to recovery faced by people with alcohol misuse.

\section{Supporting families and carers}

Little is known about formal interventions for supporting families and carers affected by the lives 
BOX 2 Challenges to recovery from alcohol misuse

Recovery can be a lengthy process and its particular challenges include:

- supporting access to specialist services (e.g. overcoming stigma)

- mobilising personal and social resources (e.g. contact with family and friends, buddying and befriending, attendance at Alcoholics Anonymous)

- changing social contacts (e.g. avoiding drinking partners)

- achieving controlled drinking, rather than abstinence

- patient's ownership of the care plan

- emotional factors (e.g. bereavement, loss, sexuality, history of abuse, relationship problems, past traumatic experiences)

- practical considerations (e.g. diet, sleep, hazardous prescribed drug interactions, physical health, drinking and driving, trip hazards, safe storage of medications)

- managing setbacks and not seeing them as failures

- managing harm reduction using the community reinforcement approach (CRA)

of older people with alcohol misuse (Copello 2002). Families often take a long time to both identify drinking problems and seek help (AdFam 2013). Fewer than 1\% of alcohol services in England provide a service specifically for older people (Wadd 2011).

Ageism from family members not appreciating the likelihood of alcohol problems in later life, together with the stigma of being labelled an 'alcoholic' and subsequent under-reporting of alcohol intake, can both influence the detection of alcohol misuse (Rockett 2006).

Concern from carers (commonly family members and friends) is the most common factor motivating older people to seek treatment for alcohol problems (Finlayson 1988). Receiving help from family and friends to cut down drinking lowers the likelihood of alcohol problems in older people (Moos 2004).

The Stress-Strain-Coping-Support (SSCS) model offers a practical approach to understanding the effects of substance misuse on family and carers (Orford 2010), with interventions centred on building resilience in individuals and a family/ social structure.

\section{Legal and ethical aspects}

Alcohol misuse in older people can present unique legal and ethical challenges. The complexity of dependence accompanied by age-related impaired decision-making results in a conflict between encouraging controlled drinking/abstinence and continuous alcohol misuse that is influenced by a lack of mental capacity. Using the core feature of harm awareness, an assessment of mental capacity in substance use can help to distinguish an unwise decision from a lack of mental capacity per se.

Capacity can also vary over time and change in relation to different decisions. In alcohol misuse, mental incapacity may fluctuate according to level of intoxication or delirium and may be associated with a revolving-door phenomenon of hospital discharge and readmission. This is further complicated by the observation that cognitive impairment may improve within the first 60 days of abstinence (Oslin 1998). Capacity should be seen as decision-specific. If a person is deemed to be 'lacking capacity', it means that they lack capacity to make a particular decision or take a particular action for themselves at the time the decision or action needs to be taken.

Although older people with alcohol-related brain damage can often be treated under the Mental Capacity Act 2005, it should be acknowledged that the Mental Health Act 1983, as amended in 2007, can also be used if there is evidence of a mental disorder such as dementia and if the criteria for using this Act are satisfied.

\section{Acute presentations of alcohol misuse}

As signs and symptoms of alcohol misuse can be very non-specific, under-reported and underrecognised, alcohol misuse may remain undetected in many patients in an acute setting.

\section{Alcohol withdrawal syndrome}

The clinical response to symptoms of alcohol withdrawal will depend on the extent of alcohol use, the degree of dependence (if any), general health and social circumstances. Treatment may include supervised care by health and social care staff or family, and home visits. However, if older people do demonstrate alcohol withdrawal syndrome, the threshold for admission to hospital may have to be lower because of the greater seriousness of medical complications such as neurological and hepatic disorder in this age group. Furthermore, a clinical judgement needs to be made about the patient's ability to make decisions about detoxification, since this may be impaired due to cognitive dysfunction directly as a result of substance misuse or indirectly as part of a co-occurring mental or physical disorder.

Although long-acting benzodiazepines are the treatment of choice for alcohol withdrawal syndrome in adults, older people should start with a lower dose. It is important to strike a balance 
between a dose sufficient to alleviate the symptoms but not enough to result in intoxication. It may be preferable to consider shorter-acting medications such as lorazepam or oxazepam, especially if there is hepatic dysfunction. It is important to ensure that the dose takes account not only of the patient's age (older people should have roughly half the dose given to a working-age adult), but also physical conditions (for example, if the patient has liver disease, accumulation may be more likely) and mental state (the patient may have a co-occurring depression, anxiety or psychosis).

An effect on memory can be detected even within the normal dose range of prescribed benzodiazepines and a dependence syndrome may result from low-dose prescription, so withdrawal with confusion may further complicate the clinical picture.

The starting dose is often related to the score on the Severity of Alcohol Dependence Questionnaire (SADQ), although this scale has been developed in younger people and should be translated cautiously. The regime consists of medication in 3 or 4 doses divided over $24 \mathrm{~h}$ and, following stabilisation over 3 or 4 days, reduction usually takes place over 7-10 days. Other prescribed and over-the-counter medication, illicit drugs and existing health conditions must be considered when formulating the dosage regime. Patients may already be on benzodiazepines or be using medications (including opiate analgesia) that are not prescribed.

Convulsions and the possibility of WernickeKorsakoff syndrome should be considered, but patients should routinely be treated with vitamins $\mathrm{B}$ and $\mathrm{C}$ by intramuscular or even intravenous administration to avoid malabsorption. Emergency treatment for anaphylaxis must be available, as although this is very rare, it can be fatal. Anti-craving agents (such as acamprosate and naltrexone) and aversive medications (such as disulfiram) should be considered, but the evidence base in older people is sparse and a clinical decision needs to be based on the condition of the patient and the adverse effects profile.

\section{Wernicke's encephalopathy}

Acute intoxication may mask the development of the potentially life-threatening Wernicke's encephalopathy, which can present during alcohol withdrawal or can be misdiagnosed as alcohol withdrawal. Wernicke's encephalopathy is a spectrum of disease resulting from thiamine deficiency, usually due to alcohol misuse. There is a greater risk in those who drink continuously rather than binge drinking, and the condition has a peak onset in men aged 40-59 and in women aged 30-49. It is usually described in terms of the classic triad of confusion, ataxia and ophthalmoplegia and is a medical emergency (Sechi 2007). It is important to maintain a high level of suspicion for the possibility of Wernicke's encephalopathy, particularly if the person is intoxicated.

The condition can be reversed if detected and treated promptly with parenteral (intramuscular or intravenous) thiamine. Untreated Wernicke's encephalopathy may lead to Korsakoff's psychosis, in which there is lasting damage to areas of the brain involved with memory (So 2012). It is important to differentiate WernickeKorsakoff syndrome from delirium and from other conditions that cause a thiamine deficiency, including thyrotoxicosis, metastatic cancer, longterm dialysis and congestive heart failure.

\section{Delirium tremens}

Delirium may be associated with intoxication or withdrawal states. Recognising delirium tremens in acute hospital settings is especially important as it has a high morbidity and mortality but is treatable. It is characterised by hallucinations, disorientation, tachycardia, hypertension, fever, agitation, convulsions and diaphoresis (sweating) and typically sets in following acute reduction/ cessation of alcohol. It typically begins 48$96 \mathrm{~h}$ after the last drink and, in the absence of complications, can last for up to 7 days (MayoSmith 1997).

Elderly patients and those with concurrent medical conditions, both acute and chronic, are at higher risk of complications. Concurrent medical conditions are common and may include dehydration, unrecognised head trauma, electrolyte abnormalities, infections (including meningitis), gastrointestinal haemorrhage, pancreatitis, liver disease and myocardial infarction. These conditions may not be obvious or self-reported in delirious patients. Close monitoring by nursing staff is critical for the patient's protection. A quiet room for rehydration and nursing is essential.

\section{Physical presentations}

Older people are more sensitive to the effects of alcohol because of reduced liver metabolism and also because the typically lower water/fat ratio in their bodies results in higher circulating levels of alcohol. Common acute physical presentations of alcohol misuse in older people are falls, memory problems and motor or sensory problems due to stroke. There is also an increased risk of subarachnoid haemorrhage. The aetiology of falls, like many of the common presentations 
described here, is multifactorial. Physiological changes associated with age, sensory deficits, postural imbalance, chronic health problems and substance misuse, and also environmental hazards, have all been identified as risk factors. Loss of balance, coordination and judgement, risk-taking, autonomic neuropathy, peripheral neuropathy, cardiac disease, osteoporosis and myopathy may all be related to alcohol misuse and make a fall more likely.

Structural and functional alcohol-related damage of the gastrointestinal system can be both acute and chronic and includes the following:

- acute gastric mucosal damage from peptic ulceration or trauma (Mallory-Weiss syndrome)

- fatty infiltration of the liver, which can progress to alcoholic hepatitis and then to cirrhosis

- weight loss from malnutrition because of reduced food intake owing to abdominal discomfort from liver damage

- portal hypertension from liver damage, associated with bleeding oesophageal varices

- increased risk of gastrointestinal cancer due to reduced levels of available vitamin $\mathrm{A}$ and the common concurrent use of tobacco.

\section{Self-harm}

Alcohol reduces anxiety and inhibitions, as well as increasing impulsivity. These factors facilitate selfharm and suicide in elderly people, who as a group are already at greater risk of suicide. Reducing suicide risk in older people with depression and alcohol misuse therefore requires both a thorough assessment and a comprehensive alcohol history.

During 2012, there were nearly 6000 suicides in the UK. The 85+ age group have the highest suicide rate, at over 20/100000 (Office for National Statistics 2014). In the under 65 population, the 'baby boomers' born between 1946 and 1964 have had higher suicide rates at any given age than earlier or later cohorts. The upper end of this cohort is already over 65 and the over-65 population will expand further over the next few decades (Conwell 2011).

The relationship between per capita alcohol consumption and suicide in older people in the UK is not as strong as in countries such as Sweden, Belgium and Portugal (Ramstedt 2001), but there are risk factors common to all age groups that need to be taken into consideration for those at risk of suicide. These often cluster together in risk profiles and they include male gender, family history of psychiatric disorder, previous attempted suicide, more severe depression, hopelessness and comorbid anxiety and alcohol misuse (Hawton 2013).
The PRISM-E study found that heavy drinking (more than $21 \mathrm{UK}$ units of alcohol per week) was positively associated with symptoms of depression and/or anxiety, as well as with perceived poor physical health (Kirchner 2007). The risk of suicide associated with alcohol dependence increases with age (Conner 2003); both mood disorder and physical illness are associated with completed suicide in older men with alcohol dependence (Conner 1999).

\section{Drug interactions}

People over 65 take more prescribed and over-thecounter drugs than younger people, and women are more likely to take these drugs than men. This means that any alcohol use may be even more problematic. Women are at particular risk of drug interactions with alcohol.

Overall, women have a lower proportion of body water (in which alcohol is distributed before it leaves the body) than men. Women have a higher blood-alcohol concentration after drinking the same amount of alcohol as men, so a woman of the same weight as a man would end up with a $50 \%$ higher blood-alcohol level (Pharmaceutical Press 2012). Alcohol also suppresses the drive to breathe, which can be fatal when taken with high doses of opiates.

The most common drugs implicated in adverse interactions with alcohol are benzodiazepines and amitriptyline (Gallagher 2008). However, antibiotics, analgesics, anti-allergy drugs, anticoagulants, anti-emetics, anticonvulsants, antihypertensives, nicotine, aspirin, paracetamol, and many other psychotropic drugs (clozapine, mirtazapine, olanzapine, quetiapine, trazodone, zuclopenthixol) all interact with alcohol.

Disulfiram is sometimes used in the treatment of alcohol dependence (under specialist supervision). It produces an unpleasant reaction after drinking just a small amount of alcohol. The reaction, which includes flushing of the face, throbbing headache, palpitations, nausea and vomiting, can last for several hours. In view of this, it is rarely used in older people.

\section{Alcohol-related brain damage}

Alcohol-related brain damage (ARBD) is a term used to cover a spectrum of disorders affecting memory, executive functioning and judgement induced by chronic and heavy alcohol consumption that results in some degree of brain damage or dysfunction. ARBD accounts for between 10 and $12.5 \%$ of cases of dementia in the UK (Lishman 1990; Harvey 1998). Only $16 \%$ of people with evidence of brain damage on 
post-mortem examination have been found to have any clinical presentation of the problem while alive (Harper 1998).

Commonly used screening instruments for cognitive function do not assess the frontal lobe, which is known to be more sensitive than other brain areas to the initial effects of alcohol toxicity (Zahr 2011). If a more comprehensive assessment of cognitive function is required, the Addenbrooke's Cognitive Examination offers such a screen (Mioshi 2006).

ARBD does not fall into any specific diagnostic classification and appropriate criteria for diagnosis have not been widely accepted, although Oslin et al (1998) defined alcohol-related dementia according to the following criteria: deterioration of memory and of one other higher cortical function, not explained by delirium, other substance misuse or alcohol withdrawal syndrome.

Short-term management should include immediate measures for detoxification and general nutrition (with use of parenteral thiamine as necessary), and treatment of any comorbid physical or psychological conditions or complications.

Long-term management depends on the person's recovery during rehabilitation. Once ARBD is established, the prognosis for recovery can be split broadly into quarters: $25 \%$ make a complete recovery, $25 \%$ make a significant recovery, 25\% make slight recovery, and $25 \%$ make no recovery (MacRae 2003). If patients make a complete or significant recovery they can be cared for in the community with the help of the family and local services, depending on the degree of disability. If they make only slight or no recovery during rehabilitation then institutional care in care homes may be the only viable option.

ARBD presents a particular challenge to recovery in older people with alcohol misuse, because those with ARBD are less likely to move to controlled drinking or abstinence than those who are cognitively intact (Rao 2013).

There remains considerable scope for service development to meet the needs of older people with ARBD (Rao 2011), particularly in the delivery of such a service within limited resources. This will require a seamless approach from health and social care professionals, policy makers, educationalists and researchers (Rao 2015b). In particular, there is a need to develop care pathways in order to improve behavioural, neuropsychological and social aspects of ARBD.

\section{Multi-agency working}

Multi-agency working is key to improving outcomes for older people with alcohol misuse (Box 3). At the level of primary care, there is an opportunity for screening and brief intervention.

BOX 3 Case history: a (fictitious) example of multi-agency working with an older person with alcohol misuse

$\mathrm{AH}$ is a 67-year-old widower referred by his general practitioner (GP) following a routine consultation. His wife had died 3 months previously. Over the past 2 months, he had escalated his alcohol intake from 4 units to 12 units per day in the form of normal-strength beer. He had also started to feel 'fed up' and had lost interest in going to the pub, drinking at home instead.

$\mathrm{AH}$ was assessed 2 weeks later on a home visit by the local community mental health team (CMHT).

At assessment, his flat was cluttered, with no food in the refrigerator and the living room was strewn with beer cans. He had been asking a neighbour to buy bread and milk, as well as alcohol. His drinking day started at around 10.00 a.m., but he did not report any morning sweats or shakes on waking.

$\mathrm{AH}$ was born in Ireland and came to live in the UK in his 20s, working mainly in the construction industry. He married in his 30 s and continued to work until he retired in his 60s. There were two children from the marriage, but they both lived abroad and there had been no contact with them since his wife died. He had had a single episode of depression in his 40 s, treated by his GP with antidepressants.
In his substance misuse history, he started drinking alcohol socially at the age of 16 , consuming 4 units of normal-strength beer every day. This drinking pattern continued until he married, when it fell to two units per day. There had been no history of marital or occupational problems associated with alcohol misuse and no history of dependence. $\mathrm{AH}$ had smoked 20 medium-tar filtered cigarettes per day since the age of 16 . There was no history of illicit drug misuse or misuse of prescribed drugs or over-the-counter medication.

In his medical history, $\mathrm{AH}$ had chronic obstructive pulmonary disease, hypertension and gastrooesophageal reflux.

A diagnosis of depressive disorder was made. He was started on a selective serotonin reuptake inhibitor and both brief and extended interventions to reduce drinking were delivered over the following 2 weeks. This was supplemented by CMHT support and attendance at a day centre for older Irish people. After successfully cutting down his alcohol intake to 2 units per day, $\mathrm{AH}$ was discharged after 6 months of CMHT support, but was re-referred 18 months later after escalating his alcohol intake following a diagnosis of diabetes mellitus. At the point of referral, he was found to show features of alcohol dependence, had stopped his antidepressant 6 months previously and was expressing suicidal thoughts.

Following a 3-month in-patient admission that involved detoxification and re-starting antidepressants, he was taken on by the older adult home treatment team and also received psychological therapy that involved motivational interviewing techniques. This was supplemented by the community reinforcement approach from community (district) nurses and medical care by the CMHT. After 12 months of CMHT support, he was discharged, again returning to controlled drinking.

Two years later, $\mathrm{AH}$ was referred by his GP following placement in a nursing home. He had reportedly developed cognitive decline and there had been several in-patient medical admissions and failed home leave (even with a package of care). At assessment, there was evidence of moderate dementia, with prominent frontal lobe impairment 
Links can also be forged with Social Services if there is a need for day care or addressing the protection of vulnerable adults. The voluntary (third) sector can also support patients, as well as their families and carers.

At the level of secondary care, there is further scope for providing services that can implement both treatment and harm reduction. For example,

- substance misuse services might offer psychological interventions (e.g. motivational interviewing) and detoxification

- old age psychiatry services might offer the community reinforcement approach (CRA) and, through their occupational therapists, can intervene to improve the quality of the living environment; they can also offer psychological treatment for comorbid mood disorders and neuropsychological testing for ARBD

- geriatric services might offer a comprehensive assessment that includes physiotherapy

- specialist housing might provide 'wet hostels' (staffed community facilities where any level of drinking is permitted, with no therapeutic focus on reducing alcohol intake) to reduce vulnerability, and there is also scope for specialised residential units that can provide an environment of controlled drinking.

The development of integrated care to manage alcohol misuse and comorbid psychiatric disorder in older people is one of the most important challenges to workforce development in the 21st century (Academy of Medical Royal Colleges 2012). Older people require a greater focus on physical disorders, depression, organic brain disorders and social factors such as social isolation, bereavement, activities of daily living, mental capacity, safeguarding and carer support.

The first UK training course for health professionals on improving knowledge, skills and attitudes regarding alcohol misuse and comorbid psychiatric disorder in older people shows promise in improving competencies in screening and brief intervention and has marked a turning point in the development of a competent workforce (Saxton 2011).

\section{Conclusions}

The number of older people is rising rapidly, with a concomitant increase in rates of comorbid mental and physical disorders, for which they inevitably take numerous prescription drugs and over-the-counter medication (Crome 2014). In this context of polypharmacy, an older person's clinical presentation can be transformed by alcohol use, misuse and dependence.
It is incumbent on medical practitioners to lead and manage teams that can assess, detect, treat and refer to relevant services. With older patients, this demands awareness of their distinctive attributes, appreciation of the complex interrelationship between addiction and other medical and psychiatric conditions, confidence in the implementation of effective interventions, a grasp of legal and ethical factors, meticulous support of teams, and perceptive appreciation of the needs of families and carers.

Detailed knowledge is needed not only of the acute effects, adverse actions and chronic consequences of alcohol use, misuse and dependence, but also of their interactions and impact on medical and mental illnesses. There is also the need to be gentle, tolerant and soothing with patients and families who are struggling with sometimes harrowing situations. A sense of hope and optimism is justified because the evidence suggests that many older people want to stop misuse and that success among people who receive treatment to curtail or contain their use is as high among older people as among younger adults. Addiction practitioners need to be aware of the special issues involved in treating older people and know how to offer an age-sensitive service, so that older people are not barred from the opportunities for improvement that are available to younger people. This involves considering the needs of older people at every point of service delivery, including availability, accessibility, intelligent adaptation of facilities and sympathetic modification of treatment options. In short, specialists can work together to value older substance misusers so that they can achieve a healthy, active future.

\section{References}

Aalto M, Alho H, Halme J, et al (2011) The Alcohol Use Disorders Identification Test (AUDIT) and its derivatives in screening for heavy drinking among the elderly. International Journal of Geriatric Psychiatry, 26: $881-5$.

Academy of Medical Royal Colleges (2012) Alcohol and Other Drugs: Core Medical Competencies (Occasional Paper OP85). Royal College of Psychiatrists.

AdFam (2013) Parental Substance Misuse: Through the Eyes of the Worker. AdFam.

Alcohol Concern (2013a) Alcohol and the Mental Health of Older People. Alcohol Concern.

Alcohol Concern (2013b) 15: 15 The Case for Better Access to Treatment for Alcohol Dependence in England. Alcohol Concern.

Blow FC, Gillespie BW, Barry KL (1998) Brief screening for alcohol problems in elderly populations using the Short Michigan Alcoholism Screening Test-Geriatric Version (SMAST-G). Alcoholism: Clinical and Experimental Research, 22 (suppl 3): 131A

Borok J, Galier P, Dinolfo M, et al (2013) Why do older unhealthy drinkers decide to make changes or not in their alcohol consumption? 
Data from the Healthy Living as You Age Study. Journal of the American Geriatrics Society, 61: 1296-302.

Conner KR, Conwell Y (1999) Age-related patterns of factors associated with completed suicide in men with alcohol dependence. American Journal on Addictions, 8: 312-8.

Conner KR, Beautrais AL, Conwell Y (2003) Moderators of the relationship between alcohol dependence and suicide and medically serious suicide attempts: analyses of Canterbury Suicide Project data. Alcoholism: Clinical and Experimental Research, 27: 1156-61.

Conwell Y, Van Orden K, Caine ED (2011) Suicide in older adults. Psychiatric Clinic of North America, 34: 451-68.

Copello A, Orford J (2002) Addiction and the family: is it time for services to take notice of the evidence? Addiction, 97: 1361-3.

Crome I, Dar K, Janikiewicz S, et al (2011) Our Invisible Addicts (College Report CR165). Royal College of Psychiatrists.

Crome I, Wu LT, Rao RT, et al (eds) (2014) Substance Use and Older People. John Wiley \& Sons.

Ewing JA (1984) Detecting alcoholism: the CAGE questionnaire. JAMA, 252: $1905-7$.

Finlayson R, Hurt R, Davis L, et al (1988) Alcoholism in elderly persons: a study of the psychiatric and psychosocial features of 216 inpatients. Mayo Clinic Proceedings, 63: 761-8.

Gallagher P, O'Mahony D (2008) STOPP (Screening Tool of Older Persons' potentially inappropriate Prescriptions): application to acutely ill elderly patients and comparison with Beers' criteria. Age and Ageing, 37: 673-9.

Harper CG, Sheedy D, Halliday GM, et al (1998) Neuropathological studies: the relationship between alcohol and aging. In Alcohol Problems and Aging (eds ESL Gomberg, AM Hegedus, RA Zucker) (NIAAA Research Monograph no. 33.): 117-34. National Institute on Alcohol Abuse and Alcoholism.

Harvey RJ, Rossor MN, Skelton-Robinson M, et al (1998) Young Onset Dementia: Epidemiology, Clinical Symptoms, Family Burden, Support and Outcome. Dementia Research Group, Imperial College London.

Hawton K, Casañas I, Comabella C, et al (2013) Risk factors for suicide in individuals with depression: a systematic review. Journal of Affective Disorders, 147: 17-28.

Kirchner JE, Zubritsky C, Cody M, et al (2007) Alcohol consumption among older adults in primary care. Journal of General Internal Medicine, 22: 92-7.

Lishman WA (1990) Alcohol and the brain. British Journal of Psychiatry, 156: 635-44.

MacRae R, Cox S (2003) Meeting the Needs of People with Alcohol Related Brain Damage: A Literature Review on the Existing and Recommended Service Provision and Models of Care. Dementia Services Development Centre, University of Stirling.

Mayo-Smith MF (1997) Pharmacological management of alcohol withdrawal: a meta-analysis and evidence-based practice guideline. JAMA, 278: 144-51.

Mioshi E, Dawson K Mitchell J et al (2006) The Addenbrooke's Cognitive Examination Revised (ACE-R): a brief cognitive test battery for dementia screening. International Journal of Geriatric Psychiatry, 21: $1078-85$

Moos RH, Schutte K, Brennan P, et al (2004) Ten-year patterns of alcohol consumption and drinking problems among older women and men. Addiction, 99: 829-38.

Office for National Statistics (2012) 2011 Census, Population and Household Estimates for the United Kingdom. ONS (http://www.ons.gov.uk/ons/publications/re-reference-tables. html?edition=tcm\%3A77-270247)

Office for National Statistics (2013) Drinking Habits Among Adults 2012. ONS (http: //www.ons.gov.uk/ons/dcp171778_338863.pdf).
Office for National Statistics (2014) Suicides in the United Kingdom, 2012 Registrations. ONS (http://www.ons.gov.uk/ons/ dcp171778_351100.pdf).

Orford J, Copello A, Velleman R, et al (2010) Family members affected by a close relative's addiction: the stress-strain-coping-support model. Drugs: Education, Prevention and Policy, 17 (suppl 1): 36-43.

Oslin D, Atkinson R, Smith D, et al (1998) Alcohol related dementia: proposed clinical criteria. International Journal of Geriatric Psychiatry, 13: 203-12.

Oslin D, Grantham S, Coakley E, et al (2006) PRISM-E: comparison of integrated care and enhanced specialty referral in managing at-risk alcohol use. Psychiatric Services, 57: 954-8.

Pharmaceutical Press (2012) Stockley's Drug Interactions. Pharmaceutical Press

Philpot M, Pearson N, Petratou V, et al (2003) Screening for problem drinking in older people referred to a mental health service: a comparison of CAGE and AUDIT. Aging \& Mental Health, 7: 171-5.

Ramstedt M (2001) Alcohol and suicide in 14 European countries. Addiction, 96: 59-75.

Rao R, Shanks A (2011) Development and implementation of a dual diagnosis strategy for older people in south east London. Advances in Dual Diagnosis, 4: 28-35.

Rao R (2013) Outcomes from liaison psychiatry referrals for older people with alcohol use disorders in the UK. Mental Health and Substance Use, 6: 362-8.

Rao RT, Crome I, Crome P, et al (2015a) Substance Misuse in Older People: An Information Guide (Cross-Faculty Report OA/AP/01). Royal College of Psychiatrists.

Rao R, Draper B (2015b) Alcohol related brain damage in older people. Lancet Psychiatry, 2: 674-45.

Roberts AME, Marshall J, Macdonald AJD (2005) Which screening test for alcohol consumption is best associated with 'at risk' drinking in older primary care attenders? Primary Care Mental Health, 3: $131-8$

Rockett IR, Putnam SL, Jia H, et al (2006) Declared and undeclared substance use among emergency department patients: a populationbased study. Addiction, 101: 706-12.

Saxton L, Lancashire S, Kipping C (2011) Meeting the training needs of staff working with older people with dual diagnosis. Advances in Dual Diagnosis, 4: 36-46.

Schonfeld L, Dupree LW (1991) Antecedents of drinking for early- and late-onset elderly alcohol abusers. Journal of Studies on Alcohol, 52 : 587-92.

Schonfeld L, King-Kallimanis BL, Duchene DM, et al (2010) Screening and brief intervention for substance misuse among older adults: the Florida BRITE project. American Journal of Public Health, 100: $108-14$

Sechi G, Serra A (2007) Wernicke's encephalopathy: new clinical settings and recent advances in diagnosis and management. Lancet Neurology, 6: 442-55.

So YT, Simon RP (2012) Deficiency diseases of the nervous system. In Bradley's Neurology in Clinical Practice (6th edn) (eds RB Daroff, GM Fenichel, J Jankovic, et al): 1340-52. Saunders.

Van den Berg JF, Kok RM, van Marwijk HW, et al (2014) Correlates of alcohol abstinence and at-risk alcohol consumption in older adults with depression: the NESDO study. American Journal of Geriatric Psychiatry, 22: 866-74.

Wadd S, Lapwort K, Sullivan M, et al (2011) Working with Older Drinkers. University of Bedfordshire.

Zahr NM, Kaufman KL, Harper CG (2011) Clinical and pathological features of alcohol-related brain damage. Nature Reviews Neurology, 7: $284-94$

\section{MCO answers \\ 1 c 2 e 3 e $4 d \quad 5$ e}




\section{MCOs}

Select the single best option for each question stem

\section{Over the past 10 years:}

a the largest decline in numbers of drinkers in any age group has been among older men drinking 8 or more units on any one day

b alcohol-related hospital admissions in the 69-74 age group have risen $20 \%$ more than in the $15-59$ age group

c alcohol-related hospital admissions of people aged 60 and over have risen by over $140 \%$

$d$ the 35-44 age group has had the highest rate of alcohol-related mortality

e of people with alcohol-related disorders, those aged 75 and over have the lowest average hospital in-patient stay.

2 Screening for alcohol misuse in older people: a involves alcohol screening tools alone in making a diagnosis b using the Alcohol Use Disorders Identification

Test (AUDIT) has the greatest validity

c can be carried out using the 1- or 3-item versions of the AUDIT

d using the Short Michigan Alcohol Screening Test-G has the poorest validity

e has greatest sensitivity and specificity for cutoff point of 4 on the AUDIT-C.

3 In older people with alcohol misuse:

a Wernicke's encephalopathy should rarely be suspected in acute intoxication

b delirium tremens in acute presentations begins 12-24 $\mathrm{h}$ after the last drink

c falls are specifically associated with postural imbalance

d there is a stronger association between per capita consumption of alcohol and suicide in the UK than in other European countries

e the 'baby boomer' cohort has higher rates of suicide than earlier or later cohorts.

\section{Adverse drug interactions with alcohol in} older people:

a are more common in men than in women

b commonly occur with aspirin

c rarely occur with benzodiazepines

d may occur with analgesics

e for disulfiram, are rarely unpleasant.

\section{Alcohol-related brain damage:}

a is confined only to a diagnosis of dementia

b rarely affects the frontal lobe

c is diagnosed if there is memory impairment alone

$\mathrm{d}$ is associated with greatest likelihood of achieving controlled drinking in ongoing alcohol misuse

e has a $25 \%$ prognosis for making a complete recovery. 